\title{
PyBioMed: a python library for various molecular representations of chemicals, proteins and DNAs and their interactions
}

\author{
Jie Dong ${ }^{1,2}$, Zhi-Jiang Yao ${ }^{1}$, Lin Zhang², Feijun Luo², Qinlu Lin², Ai-Ping Lư ${ }^{3}$, Alex F. Chen ${ }^{4}$ \\ and Dong-Sheng Cao ${ }^{1,3,4^{*}}$
}

\begin{abstract}
Background: With the increasing development of biotechnology and informatics technology, publicly available data in chemistry and biology are undergoing explosive growth. Such wealthy information in these data needs to be extracted and transformed to useful knowledge by various data mining methods. Considering the amazing rate at which data are accumulated in chemistry and biology fields, new tools that process and interpret large and complex interaction data are increasingly important. So far, there are no suitable toolkits that can effectively link the chemical and biological space in view of molecular representation. To further explore these complex data, an integrated toolkit for various molecular representation is urgently needed which could be easily integrated with data mining algorithms to start a full data analysis pipeline.

Results: Herein, the python library PyBioMed is presented, which comprises functionalities for online download for various molecular objects by providing different IDs, the pretreatment of molecular structures, the computation of various molecular descriptors for chemicals, proteins, DNAs and their interactions. PyBioMed is a feature-rich and highly customized python library used for the characterization of various complex chemical and biological molecules and interaction samples. The current version of PyBioMed could calculate 775 chemical descriptors and 19 kinds of chemical fingerprints, 9920 protein descriptors based on protein sequences, more than 6000 DNA descriptors from nucleotide sequences, and interaction descriptors from pairwise samples using three different combining strategies. Several examples and five real-life applications were provided to clearly guide the users how to use PyBioMed as an integral part of data analysis projects. By using PyBioMed, users are able to start a full pipelining from getting molecular data, pretreating molecules, molecular representation to constructing machine learning models conveniently.

Conclusion: PyBioMed provides various user-friendly and highly customized APIs to calculate various features of biological molecules and complex interaction samples conveniently, which aims at building integrated analysis pipelines from data acquisition, data checking, and descriptor calculation to modeling. PyBioMed is freely available at http:// projects.scbdd.com/pybiomed.html.
\end{abstract}

Keywords: Molecular representation, Molecular descriptors, Python library, Chemoinformatics, Data integration, Bioinformatics

\footnotetext{
*Correspondence: oriental-cds@163.com

${ }^{1}$ Xiangya School of Pharmaceutical Sciences, Central South University,

No. 172, Tongzipo Road, Yuelu District, Changsha, People's Republic

of China

Full list of author information is available at the end of the article
} 


\section{Background}

With the increasing development of biotechnology and informatics technology, the past decade has seen an exceptional growth in publicly available data in chemistry and biology, especially in human-specific molecular interaction data. The heterogeneity of data in databases poses a significant challenge to their integration and analysis in practice $[1,2]$. However, the bioinformatics and the cheminformatics communities have evolved more or less independently, e.g., with an emphasis on macro biomolecules and chemical compounds, respectively. Investigation of interactions is a complex molecular recognition process, which is not only related to the bioinformatics projects that aim at a systematic analysis of the structure and function of proteins and DNAs that scales to the genome level, but also to the chemoinformatics projects that are devoted to the analysis of structure and biological activity of chemicals. More importantly, systematic investigation of generated knowledge in both the chemical and biological knowledge spaces is required, especially in the scenarios of identifying both new targets and their potential ligands, discovering potential biomarkers for complex diseases, understanding the mechanism of interactions, and discovering new regulatory mechanism etc. [3-8]. Therefore, it is very necessary to build informatics platforms for unified data or knowledge representation that can integrate the existing efforts from both communities.

Furthermore, wealthy information in these data needs to be extracted and then transformed to useful knowledge by various data mining and artificial intelligent methods. Lots of machine learning methods have been elaborately developed to mine useful biomedicine information [9-16]. However, in order to apply various machine learning approaches on molecular data, it is a common practice to encode molecular information as numerical features. The type of encoding, however, can significantly affect analyses, and choosing a precise and effective encoding is a critical step. Molecular descriptors are one of the most powerful approaches to characterize the biological, physical, and chemical properties of molecules and have long been used in various studies for understanding molecular interactions or drug discovery. These descriptors capture and magnify distinct aspects of molecular topology in order to investigate how molecular structure affects molecular property. Molecular features have frequently been used in the development of machine learning in QSAR/QSPR [17, 18], virtual screening [19], similarity search [20], drug absorption, distribution, metabolism, elimination and toxicity (ADMET) eavaluation [21-24], protein structural and functional classes $[25,26]$, protein-protein interactions [27], compoundprotein interactions [28-31], subcellular locations and peptides of specific properties [32], meiotic recombination hot spots [33], nucleosome positioning in genomes and other drug discovery processes [34]. In terms of molecular representation importance, some web servers and stand-alone programs, such as RDKit [35], CDK [36], $r c d k, P a D E L$ [37], Cinfony [38], Chemopy [39], ChemDes [40], BioJava [41], BioTriangle [42], bioclipse [43], propy [44], PyDPI [45], Biopython, repDNA [46], CDK-Taverna [47], protr/protrWeb [48], ChemmineR [49], and Rcpi [50] have been established to calculate such descriptors. However, currently available solutions are often limited to certain types of descriptors, lack flexibility, and usually difficult to seamlessly integrate into the predictive modeling pipeline. We still urgently need a comprehensive and flexible toolkit to integrate these separate functionalities into a uniform framework, and further enable us to build a full analysis pipeline.

Here, we developed PyBioMed, a python library, for realizing comprehensive molecular representation of various molecular objects and basic data analysis tasks. PyBioMed mainly focuses on the study of molecular representation techniques for not only single molecular objects, but also interactions between any two individual molecules from pairwise samples. To easily use the $P y B i$ oMed utilities and functionalities, we provide a uniform interface and highly customized modules in the library to perform data analysis. To introduce and describe PyBioMed utility and application, we selected five wide applications as examples to show that PyBioMed can be used as an integral part of an analytical pipeline. Our computational algorithms are extensively tested and the computed features have also been used in a number of published studies. We recommend PyBioMed to analyze and represent various complex molecular data under investigation. Further, we hope that the library will be incorporated to other research projects such as data analysis pipeline, web server and software applications, in which the molecular representation will play an important role.

\section{Implementation}

The library PyBioMed is implemented in python. It is mainly based on the RDKit and pybel implementation, licensed under BSD 3-Clause License, and depends on the following python modules: scipy, numpy, os and sys. The library is mainly divided into six highly customized modules, namely: (1) PyGetMol, (2) PyPretreat, (3) PyMolecule, (4) PyProtein, (5) PyDNA and (6) PyInteraction. The modules allow the users to get various molecular objects by different ways, pretreat these molecular objects, and finally calculate chemical descriptors, protein descriptors, DNA descriptors and interaction descriptors by different functionalities. The implementation of these 
six modules is independent, and each module could perform the specific function. Additionally, four additional files include examples and applications, test modules and help documentations, which will greatly facilitate the use of the library. The PyBioMed library is freely available from the home page of the PyBioMed project. URL: http://projects.scbdd.com/pybiomed.html. The formatted documentation of the library is designed based on the sphinx language. The documentation of 149 pages includes the introduction of the library, the installation of the library, how to use each function or module by an example, five real-life applications widely covering different study fields, the detailed documentation of PyBioMed APIs, how to test the library, and the download links for detailed descriptor introduction. The users could learn all knowledge of the library by looking up the detailed documentation. The main functionalities of the library are presented in the following subsections.

\section{Downloading and reading molecular files}

Before various studies, it is the first step for the researchers to conveniently obtain various molecular objects from various web sources such as chemical structures, protein sequences, and DNA sequences. The PyBioMed library designed a specific module called PyGetMol to realize the aim. The PyGetMol module is responsible for obtaining and reading various molecular objects, and it contains three functions for downloading molecular objects (Getmol, GetProtein, GetDNA) and various functions for reading molecular objects. The Getmol module provides multiple tools to get molecular structures by the molecular ID from websites including NCBI, EBI, CAS, KEGG and DrugBank. The GetProtein module provides the tool to get protein sequence by the PDB ID and UniProt ID. The GetDNA module provides the tool to get DNA sequence by the Gene ID from websites. Additionally, they are also responsible for reading various molecular objects in different formats, such as SDF, MOL, InChI and SMILES for chemicals, FASTA for proteins and DNAs. The incorporation of these functionalities makes PyBioMed easily accessible in various cheminformatics applications.

\section{Pretreating molecular objects}

The check and preprocessing for various molecular objects is of high importance for subsequent descriptor calculation and data analysis, especially those molecular objects from web sources. Some molecules may contain structure defects to a certain extent, and therefore seriously influence or even destroy the subsequent descriptor calculation. The PyPretreat module in the library provides three specific functions which are responsible for pretreating three different molecular objects. The user could check the protein and DNA sequences by PyPretreatPro and PyPretreatDNA, respectively. The two functions mainly check whether there are additional amino acids types or DNA basic group types or not. If the sequence is right, the result goes back to the number of amino acids and nucleotides or not, otherwise, the result is 0 . For chemical compounds, the pretreating step seems to be complex. PyPretreatMol pretreats a chemical structure in the following ways: (1) normalization of functional groups to a consistent format; (2) recombination of separated charges; (3) breaking of bonds to metal atoms; (4) competitive deionization to ensure strongest acids ionize first in partially ionize molecules; (5) tautomer enumeration and canonicalization; (6) neutralization of charges; (7) standardization or removal of stereochemistry information; (8) filtering of salt and solvent fragments; (9) generation of fragment, isotope, charge, tautomer or stereochemistry insensitive parent structures; (10) validations to identify molecules with unusual and potentially troublesome characteristics. The functionalities can be highly customized by setting corresponding parameters according to the job demand. Alternatively, the users could also pretreat the molecular structure using all functions by StandardSmi function.

Calculating descriptors form chemicals, proteins and DNAs Molecular representation is the core feature of the $\mathrm{PyBi}$ oMed library. "The molecular descriptor is the final result of a logic and mathematical procedure which transforms chemical or biological information encoded within a symbolic representation of a molecule into a useful number or the result of some standardized experiment' [51]. Molecular representation plays a fundamental role in chemoinforamtics and bioinformatics, and lies at the heart of ligand-based drug design. It is the first even the most key step in the data analysis tasks. A large number of molecular descriptors from chemicals, proteins and DNAs could be easily calculated by the PyBioMed library, which covers wide applications in various cheminformatics and bioinformatics projects.

\section{Molecular descriptors based on chemical structures} The PyMolecule module in PyBioMed is responsible for calculating the commonly used structural and physicochemical descriptors. It computes twelve feature groups composed of fourteen descriptors that include 775 descriptor values. These descriptors capture and magnify distinct aspects of chemical structures, including constitutional descriptors, topological descriptors, kappa shape indices, connectivity indices, Burden descriptors, E-state indices, charge descriptors, Basak information indices, autocorrelation descriptors, molecular properties, MOE-type descriptors, and pharmacophore descriptors. 
A detailed list of descriptors for chemicals covered by PyBioMed is summarized in Table 1. The usefulness of molecular descriptors in the representation of molecular information is reflected in their widespread adoption and use across a broad range of applications and methodologies, as reported in a large number of published articles $[18,22,52,53]$. More detailed description and references can be found in the documentation of PyBioMed. We could import the corresponding module to calculate the molecular descriptors as need. Alternatively, an easier way to compute these descriptors is construct a PyMolecule object, which encapsulates all methods for the calculation of descriptors.

Besides molecular descriptors, PyBioMed also realizes the computation of a number of molecular fingerprints, and a specific fingerprint module is designed to achieve the aim. Molecular fingerprints are string representations of chemical structures, which consist of bins, each bin being a substructure descriptor associated with a specific molecular feature. 19 types of molecular fingerprints and substructure fragments are provided in PyBioMed, including topological fingerprints, E-state fingerprints, MACCS keys, FP4 keys, atom pairs fingerprints, PubChem fingerprints, topological torsion fingerprints, and Morgan/circular fingerprints etc. (see Table 2). The usefulness of these molecular fingerprints covered by PyBioMed have been sufficiently

\begin{tabular}{|c|c|c|}
\hline Feature group & Features & Number of descriptors \\
\hline Constitution & $\begin{array}{l}\text { Molecular constitutional } \\
\text { descriptors }\end{array}$ & 30 \\
\hline Topology & Topological descriptors & 35 \\
\hline Connectivity & $\begin{array}{l}\text { Molecular connectivity } \\
\text { indices }\end{array}$ & 44 \\
\hline E-state & E-state descriptors & 237 \\
\hline Bask & Bask descriptors & 21 \\
\hline Burden & Burden descriptors & 64 \\
\hline Kappa & Kappa shape descriptors & 7 \\
\hline \multirow[t]{3}{*}{ Autocorrelation } & $\begin{array}{l}\text { Moreau-Broto autocorrela- } \\
\text { tion }\end{array}$ & 32 \\
\hline & Moran autocorrelation & 32 \\
\hline & Geary autocorrelation & 32 \\
\hline Charge & Charge descriptors & 25 \\
\hline Property & Molecular property & 6 \\
\hline MOE-type & MOE-type descriptors & 60 \\
\hline Pharmacophore & $\begin{array}{l}\text { Chemically advanced tem- } \\
\text { plate search (CATS) }\end{array}$ & 150 \\
\hline
\end{tabular}

demonstrated by a number of published studies of the development of machine learning classification systems in QSAR/SAR, drug ADME/T prediction, similarity searching, clustering, ranking and classification [54].

\section{Protein or peptide descriptors based on amino acid sequences}

The PyProtein module in PyBioMed is responsible for calculating the widely used structural and physicochemical features of proteins and peptides from amino acid sequences. It computes five feature groups composed of fourteen features, including amino acid composition, dipeptide composition, tripeptide composition, normalized Moreau-Broto autocorrelation, Moran autocorrelation, Geary autocorrelation, sequence-order-coupling number, quasi-sequence-order descriptors, composition, transition and distribution of various structural and physicochemical properties, and two types of pseudo amino acid composition (PseAAC) descriptors. These features could be generally regarded as different Chou's PseAAC modes. In addition, it can also easily compute previous descriptors based on user-defined properties, which are automatically available from the AAindex database. A list of features for proteins and peptides covered by $\mathrm{PyBi}$ oMed is summarized in Table 3. These features have been used for predicting protein- and peptide-related problems by using machine learning methods. More detailed description and references can be found in the documentation of PyBioMed.

In fact, the abovementioned features can be regarded as different Chou's PseAAC modes [55]. For example, amino acid, dipeptide, tripeptide, or $k$-mer peptide $(k=4,5, \ldots)$ compositions are just different modes of Chou's PseAAC. Moreover, the higher-level features such as GO (Gene Ontology) information, FunD (Functional Domain) information, and Sequential Evolution information are also skillfully fused into the Chou's PseAAC descriptors to characterize different protein information which is widely used for solving various biological problems. An excellent review by Chou has pointed out their relevancy.

\section{DNA descriptors based on nucleotide sequences}

The PyDNA module in PyBioMed is responsible for calculating the widely used structural and physicochemical features of DNAs from nucleotide sequences. Generally, three groups of features from nucleotide sequences are calculated to represent DNA in PyBioMed. (1) three nucleic acid composition features describing the local sequence information by means of $k$-mers (subsequences of DNA sequences); (2) six autocorrelation features 
Table 2 Molecular fingerprints of chemicals calculated by PyBioMed

\begin{tabular}{|c|c|c|}
\hline Feature group & Features & Number of descriptors \\
\hline \multirow[t]{6}{*}{ Substructure-based fingerprints } & MACCS fingerprints & 166 \\
\hline & E-state fingerprints & 79 \\
\hline & Ghose-Crippen fingerprints & 110 \\
\hline & FP3 fingerprints & 210 \\
\hline & FP4 fingerprints & 307 \\
\hline & PubChem fingerprints & 881 \\
\hline \multirow[t]{13}{*}{ Fingerprints } & Daylight-type fingerprints & 2048 \\
\hline & Atom pairs fingerprints & 2048 \\
\hline & Topological torsion fingerprints & 2048 \\
\hline & FP2 fingerprints & 1024 \\
\hline & ECFP2 fingerprints & 1024 \\
\hline & ECFP4 fingerprints & 1024 \\
\hline & ECFP6 fingerprints & 1024 \\
\hline & FCFP2 fingerprints & 1024 \\
\hline & FCFP4 fingerprints & 1024 \\
\hline & FCFP6 fingerprints & 1024 \\
\hline & Morgan fingerprints & 1024 \\
\hline & Pharm2D2point fingerprints & 135 \\
\hline & Pharm2D3point fingerprints & 2135 \\
\hline
\end{tabular}

Table 3 Protein descriptors of proteins or peptides calculated by PyBioMed

\begin{tabular}{|c|c|c|}
\hline Feature group & Features & Number of descriptors \\
\hline \multirow[t]{3}{*}{ Amino acid composition } & Amino acid composition & 20 \\
\hline & Dipeptide composition & 400 \\
\hline & Tripeptide composition & 8000 \\
\hline \multirow[t]{3}{*}{ Autocorrelation } & Normalized Moreau-Broto autocorrelation & $240^{a}$ \\
\hline & Moran autocorrelation & $240^{a}$ \\
\hline & Geary autocorrelation & $240^{a}$ \\
\hline \multirow[t]{3}{*}{ CTD } & Composition & 21 \\
\hline & Transition & 21 \\
\hline & Distribution & 105 \\
\hline Conjoint triad & Conjoint triad features & 343 \\
\hline \multirow[t]{2}{*}{ Quasi-sequence order } & Sequence order coupling number & 60 \\
\hline & Quasi-sequence order descriptors & 100 \\
\hline \multirow[t]{2}{*}{ Pseudo amino acid composition } & Pseudo amino acid composition & $50^{b}$ \\
\hline & Amphiphilic pseudo amino acid composition & $50^{c}$ \\
\hline
\end{tabular}

a The number depends on the choice of the number of properties of amino acid and the choice of the maximum values of the lag. The default is use eight types of properties and $l a g=30$

${ }^{b}$ The number depends on the choice of the number of the set of amino acid properties and the choice of the $\lambda$ value. The default is use three types of properties proposed by Chou et al. and $\lambda=30$

c The number depends on the choice of the $\lambda$ value. The default is that $\lambda=15$ 
describing the level of correlation between two oligonucleotides along a DNA sequence in terms of their specific physicochemical properties; (3) six pseudo nucleotide composition features, which can be used to represent a DNA sequence with a discrete model or vector yet still keep considerable sequence order information, particularly the global or long-range sequence order information, via the physicochemical properties of its constituent oligonucleotides. A detailed list of descriptors for DNAs covered by PyBioMed is summarized in Table 4. The usefulness of these features covered by PyBioMed for representing DNA sequence information have been sufficiently demonstrated by a number of published studies in computational genomics and genome sequence analysis. More detailed description and references can be found in the documentation of the PyBioMed library. There are two ways to calculate DNA descriptors in the $P y D N A$ module. One is to directly use the corresponding methods, the other one is firstly to construct a PyDNA class and then run their methods to obtain the descriptors. The users could select one or more groups to represent DNAs under investigation. It should be noted that the output is a dictionary form, whose keys and values represent the descriptor name and the descriptor value, respectively. The user could clearly understand the meaning of each descriptor.

\section{Interaction descriptors based on pairwise samples Descriptors from the interaction between two molecules with the same type}

The interaction between two molecules with the same type includes drug-drug interaction, protein-protein interaction, etc. However, the construction process of the interaction descriptors of them is similar to each other. We will show how to construct an interaction feature by the protein-protein interaction example. Let $\mathbf{F}_{\mathrm{a}}=\left\{\mathbf{F}_{\mathbf{a}}(\mathrm{i})\right.$, $\mathrm{i}=1,2, \ldots, \mathrm{p}\}$ and $\mathbf{F}_{\mathbf{b}}=\left\{\mathbf{F}_{\mathbf{b}}(\mathrm{i}), \mathrm{i}=1,2, \ldots, \mathrm{p}\right\}$ are the two descriptor vectors for interaction protein $A$ and protein $B$, respectively. There are three methods to construct the interaction descriptor vector $\mathbf{F}$ for $\mathrm{A}$ and $\mathrm{B}$ :

1. Two vectors $\mathbf{F}_{\mathbf{a b}}$ and $\mathbf{F}_{\mathbf{b a}}$ with dimension of $2 p$ are constructed: $\mathbf{F}_{\mathrm{ab}}=\left(\mathbf{F}_{\mathrm{a}}, \mathbf{F}_{\mathbf{b}}\right)$ for interaction between protein $A$ and protein $B$ and $\mathbf{F}_{\mathbf{b a}}=\left(\mathbf{F}_{\mathbf{b}}, \mathbf{F}_{\mathbf{a}}\right)$ for interaction between protein $B$ and protein $A$.

2. One vector $\mathbf{F}$ with dimension of $2 p$ is constructed: $\mathbf{F}=\left\{\mathbf{F}_{\mathbf{a}}(\mathrm{i})+\mathbf{F}_{\mathbf{b}}(\mathrm{i}), \mathbf{F}_{\mathbf{a}}(\mathrm{i}) \times \mathbf{F}_{\mathbf{b}}(\mathrm{i}), \mathrm{i}=1,2, \ldots, \mathrm{p}\right\}$.

3. One vector $\mathbf{F}$ with dimension of $p^{2}$ is constructed by the tensor product: $\mathbf{F}=\left\{\mathbf{F}(\mathrm{k})=\mathbf{F}_{\mathbf{a}}(\mathrm{i}) \times \mathbf{F}_{\mathbf{b}}(\mathrm{j}), \mathrm{i}=1\right.$, $2, \ldots, p, j=1,2, \ldots, p, k=(i-1) \times p+j\}$.

\section{Descriptors from the interaction between two molecules with different types}

The interaction between the molecules with different types includes chemical-protein interaction, proteinDNA interaction, and chemical-DNA interaction. However, the calculation of these interaction descriptors is similar to each other. Likewise, we will show how to construct an interaction feature by the chemical-protein interaction example. There are two methods for construction of descriptor vector $\mathbf{F}$ for chemical-protein interaction from the protein descriptor vector $\mathbf{F}_{\mathbf{t}}\left(\mathbf{F}_{\mathbf{t}}(\mathrm{i})\right.$, $\left.\mathrm{i}=1,2, \ldots, \mathrm{p}_{\mathrm{t}}\right)$ and chemical descriptor vector $\mathbf{F}_{\mathbf{d}}\left(\mathbf{F}_{\mathbf{d}}(\mathrm{i})\right.$, $\left.\mathrm{i}=1,2, \ldots, \mathrm{p}_{\mathrm{d}}\right)$ :

Table 4 DNA descriptors of DNAs calculated by PyBioMed

\begin{tabular}{|c|c|c|}
\hline Feature group & Features & Number of descriptors ${ }^{a}$ \\
\hline \multirow[t]{2}{*}{ Nucleic acid composition } & Basic kmer & 16 \\
\hline & Reverse compliment kmer & 12 \\
\hline \multirow[t]{6}{*}{ Autocorrelation } & Dinucleotide-based auto covariance & 76 \\
\hline & Dinucleotide-based cross covariance & 2812 \\
\hline & Dinucleotide-based auto-cross covariance & 2888 \\
\hline & Trinucleotide-based auto covariance & 24 \\
\hline & Trinucleotide-based cross covariance & 264 \\
\hline & Trinucleotide-based auto-cross covariance & 288 \\
\hline \multirow[t]{6}{*}{ Pseudo nucleic acid composition } & Pseudo dinucleotide composition & 18 \\
\hline & Pseudo k-tuple nucleotide composition & 18 \\
\hline & Parallel correlation pseudo dinucleotide composition & 18 \\
\hline & Parallel correlation pseudo trinucleotide composition & 66 \\
\hline & Series correlation pseudo dinucleotide composition & 90 \\
\hline & Series correlation pseudo trinucleotide composition & 88 \\
\hline
\end{tabular}

\footnotetext{
a The number depends on the choice of the values of the parameters in the formula. Here, the number of each type of descriptors is based on the default parameter
} value. For detailed information, please refer to the documentation section in the PyBioMed manual 
1. One vector $\mathbf{V}$ with dimension of $\mathrm{p}_{t}+\mathrm{p}_{\mathrm{d}}$ is constructed: $\mathbf{F}=\left(\mathbf{F}_{\mathbf{t}}, \mathbf{F}_{\mathbf{d}}\right)$ for interaction between protein $t$ and chemical $d$.

2. One vector $\mathbf{V}$ with dimension of $\mathrm{p}_{t} \times \mathrm{p}_{\mathrm{d}}$ is constructed by the tensor product: $\mathbf{F}=\left\{\mathbf{F}(\mathrm{k})=\mathbf{F}_{\mathbf{t}}(\mathrm{i}) \times \mathbf{F}_{\mathbf{d}}(\mathrm{j}), \mathrm{i}=1\right.$, $\left.2, \ldots, \mathrm{p}_{\mathrm{t}}, \mathrm{j}=1,2, \ldots, \mathrm{p}_{\mathrm{d}}, \mathrm{k}=(\mathrm{i}-1) \times \mathrm{p}_{\mathrm{t}}+\mathrm{j}\right\}$.

\section{How to use PyBioMed functions}

In order to familiarize the users with PyBioMed, It is recommended that the user works through the tutorial examples provided. The tutorial will go through the process of installing and running an example in some detail. The PyBioMed library would be applied to solve various research tasks in the field of cheminformatics, bioinformatics and systems biology. We introduced five examples of its applications in the documentation including Caco-2 cell permeability, aqueous solubility, drug-target interaction data, protein subcellular location, and nucleosome positioning in genomes. Next, we will briefly introduce the installation of PyBioMed, and how to calculate molecular descriptors by writing few lines of codes.

PyBioMed has been successfully tested on Linux and Windows systems. The installation process of PyBioMed is very easy. However, the user first needs to install RDKit and pybel successfully. The detailed installation steps are described in the README page of the GitHub repository of PyBioMed.

There are two means to compute these molecular descriptors from small molecules. One is to use the builtin modules. We could import related functions to compute these features. For example, the topology module includes a number of functionalities used for calculating various topological descriptors. The user could conveniently use them as need.

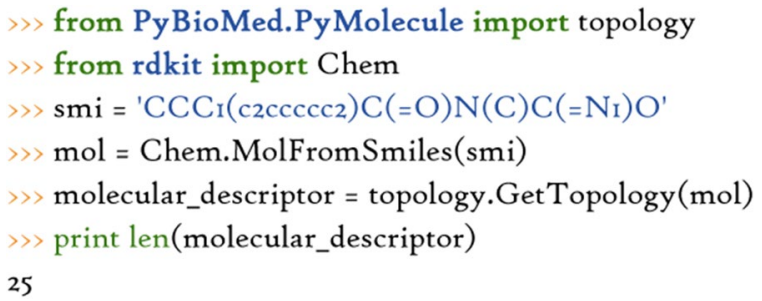

The other is to call the related class by importing the class module, which encapsulates commonly used descriptor calculation methods. Pymolecule, Pyprotein and Pydna are responsible for the calculation of chemical descriptors, protein descriptors, and DNA descriptors, respectively. We could construct a corresponding object with a molecule input, and then call corresponding methods to calculate these features.

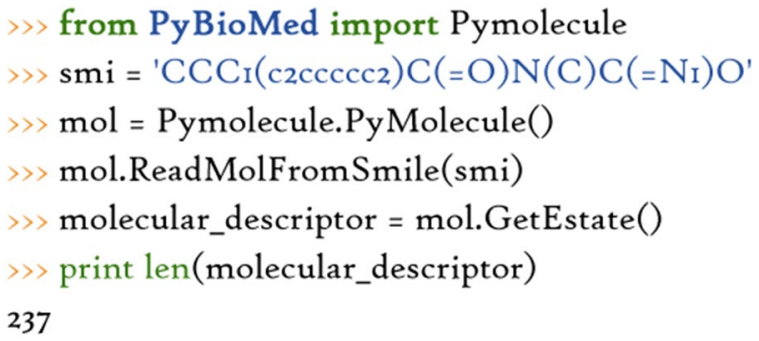

The interaction descriptors for pairwise samples are calculated as follows:

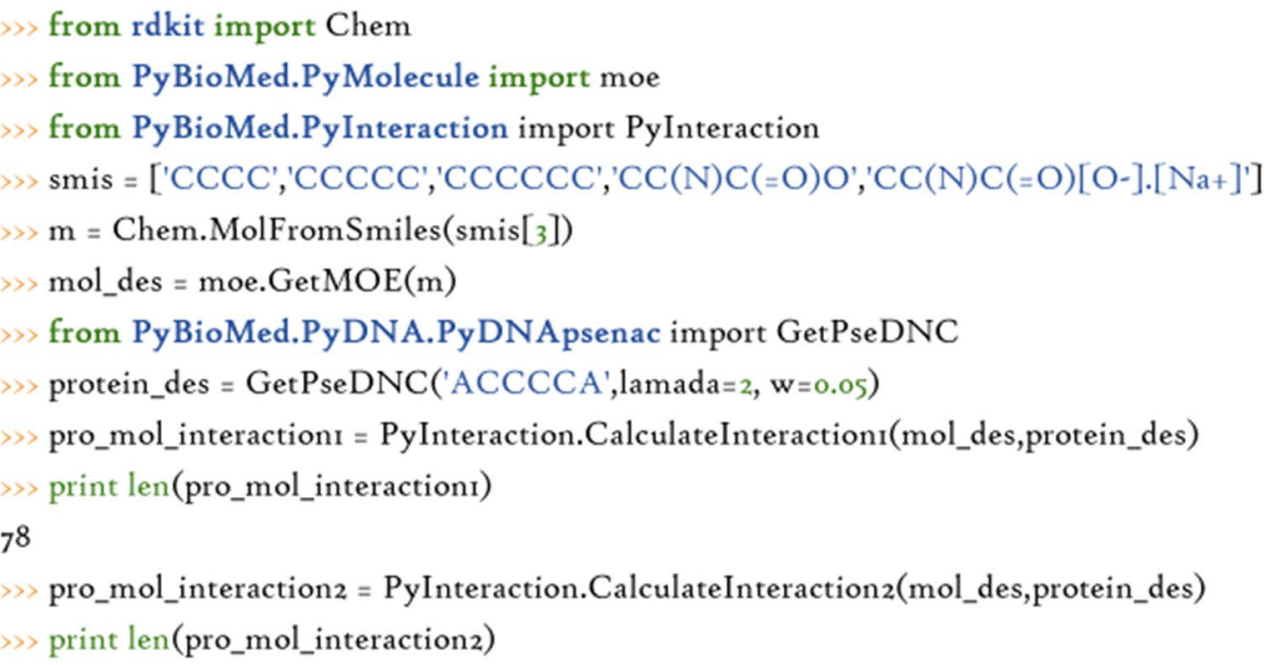




\section{Discussion}

Considering the amazing rate at which data are accumulated in chemistry and biology fields, new tools that process and interpret large and complex interaction data are increasingly important. However, to our knowledge, no open source or freely available tool exists to perform all these functions above in a uniform framework. PyBioMed is a powerful python library for the extraction of features of complex interaction data. After representation, different statistical learning tools can be applied for further analysis and visualization of the data. Several case studies from wide applications show how PyBioMed was used to describe various molecular features and establish a model in a routing way (See documentation). The application domain of PyBioMed is not limited to the specific data type. It can, as Fig. 1 shows, be applied to a broad range of scientific fields such as QSAR/SAR, similarity search, virtual screening, ADMET prediction, ligandbased drug discovery, protein function/substructure/ family classification, subcellular locations, post-translational modification (PTM), DNA structure/function/site prediction, and various interaction data analysis such as drug-target/drug interaction and protein-protein interaction studies. We expect that PyBioMed will better assist chemists, pharmacologists and biologists in characterizing, analyzing, and comparing complex molecular objects.

As mentioned in the background, there are several tools that have been developed to calculate chemical, protein or DNA/RNA description. However, PyBioMed is unique because it can be used to extract features of complex interaction data. Commonly, these tools can be divided into three types: software production, software package and web server. Software production tends to be commercial; web server tries to provide user-friendly way to beginners or users without programming skills; software package provides most flexible way to calculate descriptors in different application scenarios. The three types of tools have their own advantages. Here, PyBioMed belongs to software package of Python community. In order to give a more clear insight into the different software packages, we made a comparison between them (see Table 5).

From the Table 5, we can see: (1) most of these tools can only realize one or two functionalities while $P y B i$ oMed made all these functionalities into a uniform framework. (2) For some tools, the implications are actually not the same though the same functionality is ticked. Both RDKit and PyBioMed have the function of 'Pretreating molecules', while PyBioMed reorganized and wrapped the basic functions of RDKit into 10 new functions. By combining these functions in different orders and setting the corresponding parameters, users can realize the customized pretreating process. The specific functions for pretreating molecules and calculating protein descriptors of BioJava and PyBioMed are completely different. ChemminR can retrieve molecules from PubChem database while PyBioMed can retrieve molecules from NCBI, CAS, KEGG databases. ChemminR can calculate some basic molecular properties while PyBioMed can calculate various and diverse molecular descriptors, especially a series of featured fingerprints. PyDPI is able to calculate interaction descriptors but only limited to chemical-protein interaction feature. PyBioMed realized to represent nine types of interaction features. (3) The application scenarios are not the same though some tools can calculate the same kinds of descriptors. For example, BioJava is for Java environment, and ChemminR is for R environment, while PyBioMed is designed for Python environment. In summary, PyBioMed is a different tool from them and

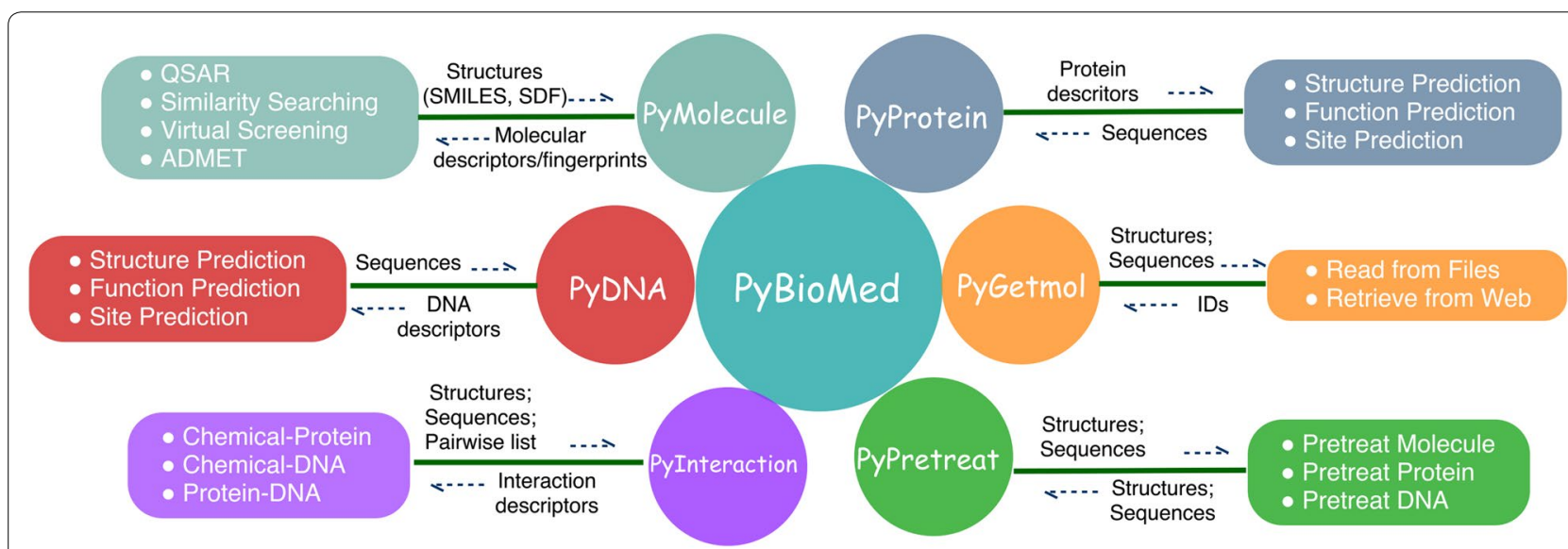

Fig. 1 The main modules of the PyBioMed library and their corresponding wide applications in chemoinformatics, bioinformatics and drug discovery process 
Table 5 Differences of software packages for descriptor calculation

\begin{tabular}{lllllll}
\hline Tool names & $\begin{array}{l}\text { Retrieving mol- } \\
\text { ecules }\end{array}$ & $\begin{array}{l}\text { Pretreating mol- } \\
\text { ecules }\end{array}$ & $\begin{array}{l}\text { Chemical descrip- } \\
\text { tors/fingerprints }\end{array}$ & $\begin{array}{l}\text { Protein descriptors } \\
\text { DNA/RNA descrip- }\end{array}$ & $\begin{array}{l}\text { Interaction } \\
\text { descriptors }\end{array}$ \\
\hline PyBioMed & $\sqrt{ }$ & $\sqrt{ }$ & $\sqrt{ }$ & $\sqrt{ }$ & $\sqrt{ }$ & $\sqrt{ }$ \\
PyDPI & & & $\sqrt{ }$ & & $\sqrt{ }$ \\
ChemoPy & & $\sqrt{ }$ & $\sqrt{ }$ & & \\
Cinfony & & $\sqrt{ }$ & & \\
RDKit & & $\sqrt{ }$ & & \\
CDK & & $\sqrt{ }$ & $\sqrt{ }$ \\
rcdk & & $\sqrt{ }$ & $\sqrt{ }$ \\
PaDEL & & $\sqrt{ }$ & $\sqrt{ }$ \\
BioJava & $\sqrt{ }$ & $\sqrt{ }$ & \\
Rcpi & & $\sqrt{ }$ & & $\sqrt{ }$ \\
ChemmineR & $\sqrt{ }$ & & & \\
Propy & & & & \\
RepDNA & & & & \\
\hline
\end{tabular}

provides lots of unique functionalities, especially when extracting features of complex interaction data and realizing a full data analysis pipeline.

\section{Conclusion}

PyBioMed provides a freely available and ease-to-use python library to calculate various features of biological molecules and complex interaction samples conveniently. It makes a step in this direction providing a way to fully integrate information from chemical space and biology space into an interaction space, which cannot be performed by other existing tools. As far as we know, PyBioMed is the first python library that calculates both individual descriptors of three types of molecular objects and any interactions between two of them in a uniform framework. It provides not only the detailed information about all descriptors and how to calculate them but also several tutorials and corresponding model scripts for different applications. In addition, the functions and modules related in PyBioMed and the stability of the library was extensively tested. We hope that the library will be helpful when exploring questions concerning structures, functions and interactions of various molecular data in the context of chemoinformatics. We also expect that our/other groups may use the free code of PyBioMed and the new machine learning models to implement public web servers. The increasingly diversified and further applications of molecular descriptors urge new descriptors and new tools to be developed, and researches have achieved some results [56-59]. In future work, we plan to apply the integrated features on various biological research questions, and to extend the range of functions with new promising descriptors for the coming versions of the library.

\section{Authors' contributions}

JD, ZJY and DSC wrote the code; JD, ZJY and DSC released the package and manual; DSC, AFC and APL wrote and checked the manuscript. FJL, QLL and $\mathrm{LZ}$ helped in preparing figures and tables, testing and validating the results. FJL, QLL, AFC and APL helped in giving suggestions to improve the software package. All authors contributed into design of the study. All authors read and approved the final manuscript.

\section{Author details}

${ }^{1}$ Xiangya School of Pharmaceutical Sciences, Central South University, No. 172, Tongzipo Road, Yuelu District, Changsha, People's Republic of China.

${ }^{2}$ College of Food Science and Engineering, National Engineering Laboratory for Deep Processing of Rice and Byproducts, Central South University of Forestry and Technology, Changsha, China. ${ }^{3}$ Institute for Advancing Translational Medicine in Bone and Joint Diseases, School of Chinese Medicine, Hong Kong Baptist University, Hong Kong SAR, China. ${ }^{4}$ Center for Vascular Disease and Translational Medicine, Third Xiangya Hospital, Central South University, Changsha, People's Republic of China.

\section{Competing interests}

The authors declare that they have no competing interests.

\section{Availability and requirements}

Project name: PyBioMed. Project home page: Source code is available at http:// projects.scbdd.com/pybiomed.html or https://github.com/gadsbyfly/PyBioMed. Operating system(s): Windows, Linux. Programming language: Python. License: BSD 3-Clause License. Any restrictions to use by non-academics: none.

Ethics approval and consent to participate Not applicable.

\section{Funding}

This work is financially supported by the National Natural Science Foundation of China (Grant No. 81402853 and 81501619), grants from the Project of Innovation-driven Plan in Central South University, the Postdoctoral Science Foundation of Central South University, the Chinese Postdoctoral Science Foundation (2014T70794, 2014M562142) and the Key Laboratory of Special Medicine Food Process in Hunan Province (2017TP1021). The studies meet with the approval of the university's review board.

\section{Publisher's Note}

Springer Nature remains neutral with regard to jurisdictional claims in published maps and institutional affiliations. 
Received: 6 September 2017 Accepted: 12 March 2018 Published online: 20 March 2018

\section{References}

1. Barabasi A-L, Oltvai ZN (2004) Network biology: understanding the cell's functional organization. Nat Rev Genet 5(2):101-113

2. Barabási A-L, Gulbahce N, Loscalzo J (2011) Network medicine: a network-based approach to human disease. Nat Rev Genet 12(1):56-68

3. Cao D, Xiao N, Li Y, Zeng W, Liang Y, Lu A et al (2015) Integrating multiple evidence sources to predict adverse drug reactions based on a systems pharmacology model. CPT Pharmacometrics Syst Pharmacol 4(9):498-506

4. Yao Z, Dong J, Che Y, Zhu M, Wen M, Wang N et al (2016) TargetNet: a web service for predicting potential drug-target interaction profiling via multi-target SAR models. J Comput Aided Mol Des 30(5):413-424

5. Speck-Planche A, Kleandrova W, Luan F, Cordeiro MNDS (2013) Unified multi-target approach for the rational in silico design of anti-bladder cancer agents. Anticancer Agents Med Chem 13(5):791-800

6. Mestres J (2004) Computational chemogenomics approaches to systematic knowledge-based drug discovery. Curr Opin Drug Discov Dev 7(3):304-313

7. Perez-Nueno VI, Souchet M, Karaboga AS, Ritchie DW (2015) GESSE: predicting drug side effects from drug-target relationships. J Chem Inf Model 55(9):1804-1823

8. Napolitano F, Zhao Y, Moreira VM, Tagliaferri R, Kere J, D'Amato M et al (2013) Drug repositioning: a machine-learning approach through data integration. J Cheminform 5(1):30

9. Dong J, Yao Z-J, Zhu M-F, Wang N-N, Lu B, Chen AF et al (2017) ChemSAR: an online pipelining platform for molecular SAR modeling. J Cheminform 9(1):27

10. Ding H, Takigawa I, Mamitsuka H, Zhu S (2014) Similarity-based machine learning methods for predicting drug-target interactions: a brief review. Brief Bioinform 15(5):734-747

11. Cheng J, Tegge AN, Baldi P (2008) Machine learning methods for protein structure prediction. IEEE Rev Biomed Eng 1:41-49

12. Varnek A, Baskin I (2012) Machine learning methods for property prediction in chemoinformatics: quo vadis? J Chem Inf Model 52(6):1413-1437

13. Cortes-Ciriano I, Westen GJP, Lenselink EB, Murrell DS, Bender A, Malliavin $\mathrm{T}$ (2014) Proteochemometric modeling in a Bayesian framework. J Cheminform 6(1):35

14. Agarwal S, Dugar D, Sengupta S (2010) Ranking chemical structures for drug discovery: a new machine learning approach. J Chem Inf Model 50(5):716-731

15. Cao D-S, Liang Y-Z, Xu Q-S, Hu Q-N, Zhang L-X, Fu G-H (2011) Exploring nonlinear relationships in chemical data using kernel-based methods. Chemometr Intell Lab Syst 107(1):106-115

16. Cao D-S, Xu Q-S, Liang Y-Z, Zhang L-X, Li H-D (2010) The boosting: A new idea of building models. Chemometr Intell Lab Syst 100(1):1-11

17. Cao D-S, Xu Q-S, Liang Y-Z, Chen X, Li H-D (2010) Prediction of aqueous solubility of druglike organic compounds using partial least squares, back-propagation network and support vector machine. J Chemom 24(9):584-595

18. Wang J, Cao D, Zhu M, Yun Y, Xiao N, Liang Y (2015) In silico evaluation of $\log D(7.4)$ and comparison with other prediction methods. J Chemom 29(7):389-398

19. Geppert H, Vogt M, Bajorath J (2010) Current trends in ligand-based virtual screening: molecular representations, data mining methods, new application areas, and performance evaluation. J Chem Inf Mode 50(2):205-216

20. Willett $P(2011)$ Similarity searching using 2D structural fingerprints. Methods Mol Biol 672:133-158

21. Wang N-N, Huang C, Dong J, Yao Z-J, Zhu M-F, Deng Z-K et al (2017) Predicting human intestinal absorption with modified random forest approach: a comprehensive evaluation of molecular representation, unbalanced data, and applicability domain issues. RSC Adv 7(31):19007-19018

22. Wang N-N, Dong J, Deng Y-H, Zhu M-F, Wen M, Yao Z-J et al (2016) ADME properties evaluation in drug discovery: prediction of Caco-2 cell permeability using a combination of NSGA-II and boosting. J Chem Inf Model 56(4):763-773

23. Maltarollo VG, Gertrudes JC, Oliveira PR, Honorio KM (2015) Applying machine learning techniques for ADME-Tox prediction: a review. Expert Opin Drug Metab Toxicol 11(2):259-271

24. Wang S, Li Y, Wang J, Chen L, Zhang L, Yu H et al (2012) ADMET evaluation in drug discovery. 12. Development of binary classification models for prediction of hERG potassium channel blockage. Mol Pharm 9(4):996-1010

25. Dubchak I, Muchnik I, Holbrook SR, Kim SH (1995) Prediction of protein folding class using global description of amino acid sequence. Proc Nat Acad Sci 92(19):8700-8704

26. Huang J-H, Cao D-S, Yan J, Xu Q-S, Hu Q-N, Liang Y-Z (2012) Using core hydrophobicity to identify phosphorylation sites of human $\mathrm{G}$ proteincoupled receptors. Biochimie 94(8):1697-1704

27. Shen J, Zhang J, Luo X, Zhu W, Yu K, Chen K et al (2007) Predicting protein-protein interactions based only on sequences information. Proc Natl Acad Sci USA 104(11):4337-4341

28. Cao D-S, Liang Y-Z, Deng Z, Hu Q-N, He M, Xu Q-S et al (2013) Genomescale screening of drug-target associations relevant to Ki using a chemogenomics approach. PLoS ONE 8(4):e57680

29. Cao DS, Zhou GH, Liu S, Zhang LX, Xu QS, He M et al (2013) Largescale prediction of human kinase-inhibitor interactions using protein sequences and molecular topological structures. Anal Chim Acta 792:10-18

30. Cao D-S, Liu S, Xu Q-S, Lu H-M, Huang J-H, Hu Q-N et al (2012) Large-scale prediction of drug-target interactions using protein sequences and drug topological structures. Anal Chim Acta 752:1-10

31. Luo H, Zhang P, Huang H, Huang J, Kao E, Shi L et al (2014) DDI-CPI, a server that predicts drug-drug interactions through implementing the chemical-protein interactome. Nucleic Acids Res 42(W1):W46-W52

32. Chou K-C, Shen H-B (2008) Cell-PLoc: a package of web servers for predicting subcellular localization of proteins in various organisms. Nat Protoc 3(2):153-162

33. Chen W, Feng P-M, Lin H, Chou K-C (2013) iRSpot-PseDNC: identify recombination spots with pseudo dinucleotide composition. Nucleic Acids Res 41(6):e68

34. Guo S-H, Deng E-Z, Xu L-Q, Ding H, Lin H, Chen W et al (2014) iNucPseKNC: a sequence-based predictor for predicting nucleosome positioning in genomes with pseudo k-tuple nucleotide composition. Bioinformatics 30(11):1522-1529

35. RDKit: Open-source cheminformatics. http://www.rdkit.org. Accessed 28 Nov 2016

36. Steinbeck C, Hoppe C, Kuhn S, Floris M, Guha R, Willighagen E (2006) Recent developments of the chemistry development kit (CDK) —an open-source java library for chemo- and bioinformatics. Curr Pharm Des 12(17):2111-2120

37. Yap CW (2011) PaDEL-descriptor: an open source software to calculate molecular descriptors and fingerprints. J Comput Chem 32(7):1466-1474

38. O'Boyle N, Hutchison G (2008) Cinfony_combining open source cheminformatics toolkits behind a common interface. Chem Cent J 2(1):24

39. Cao D-S, Xu Q-S, Hu Q-N, Liang Y-Z (2013) ChemoPy: freely available python package for computational biology and chemoinformatics. Bioinformatics 29(8):1092-1094

40. Dong J, Cao D, Miao H, Liu S, Deng B, Yun Y et al (2015) ChemDes: an integrated web-based platform for molecular descriptor and fingerprint computation. J Cheminform 7(1):60

41. Holland RCG, Down TA, Pocock M, Prlia A, Huen D, James K et al (2008) BioJava: an open-source framework for bioinformatics. Bioinformatics 24(18):2096-2097

42. Dong J, Yao Z-J, Wen M, Zhu M-F, Wang N-N, Miao H-Y et al (2016) BioTriangle: a web-accessible platform for generating various molecular representations for chemicals, proteins, DNAs/RNAs and their interactions. J Cheminform 8(1):34

43. Spjuth $\mathrm{O}$, Helmus T, Willighagen EL, Kuhn S, Eklund M, Wagener J et al (2007) Bioclipse: an open source workbench for chemo- and bioinformatics. BMC Bioinform 8(1):59

44. Cao D-S, Xu Q-S, Liang Y-Z (2013) propy: a tool to generate various modes of Chou's PseAAC. Bioinformatics 29(7):960-962 
45. Cao D-S, Liang Y-Z, Yan J, Tan G-S, Xu Q-S, Liu S (2013) PyDPI: freely available Python package for chemoinformatics, bioinformatics, and chemogenomics studies. J Chem Inf Model 53(11):3086-3096

46. Liu B, Liu F, Fang L, Wang X, Chou K-C (2015) repDNA: a Python package to generate various modes of feature vectors for DNA sequences by incorporating user-defined physicochemical properties and sequenceorder effects. Bioinformatics 31(8):1307-1309

47. Kuhn T, Willighagen EL, Zielesny A, Steinbeck C (2010) CDK-Taverna: an open workflow environment for cheminformatics. BMC Bioinform 11(1):159

48. Xiao N, Cao D-S, Zhu M-F, Xu Q-S (2015) protr/ProtrWeb: R package and web server for generating various numerical representation schemes of protein sequences. Bioinformatics 31(11):1857-1859

49. Cao Y, Charisi A, Cheng L-C, Jiang T, Girke T (2008) ChemmineR: a compound mining framework for R. Bioinformatics 24(15):1733-1734

50. Cao D-S, Xiao N, Xu Q-S, Chen AF (2014) Rcpi: R/Bioconductor package to generate various descriptors of proteins, compounds, and their interactions. Bioinformatics 31(2):279-281

51. Todeschini R, Consonni V (2000) Handbook of molecular descriptors. Wiley, London

52. Dong J, Wang N-N, Liu K-Y, Zhu M-F, Yun Y-H, Zeng W-B et al (2017) ChemBCPP: a freely available web server for calculating commonly used physicochemical properties. Chemometr Intell Lab Syst 171:65-73

53. Wang NN, Deng ZK, Huang C, Dong J, Zhu MF, Yao ZJ et al (2017) ADME properties evaluation in drug discovery: prediction of plasma protein binding using NSGA-II combining PLS and consensus modeling. Chemometr Intell Lab Syst 170:84-95

54. Cao D-S, Hu Q-N, Xu Q-S, Yang Y-N, Zhao J-C, Lu H-M et al (2011) In silico classification of human maximum recommended daily dose based on modified random forest and substructure fingerprint. Anal Chim Acta 692(1-2):50-56

55. Chou K-C (2011) Some remarks on protein attribute prediction and pseudo amino acid composition. J Theor Biol 273(1):236-247

56. Garcia-Jacas CR, Marrero-Ponce Y, Acevedo-Martinez L, Barigye SJ, Valdes-Martini JR, Contreras-Torres E (2014) QuBiLS-MIDAS: a parallel freesoftware for molecular descriptors computation based on multilinear algebraic maps. J Comput Chem 35(18):1395-1409

57. Valdes-Martini JR, Marrero-Ponce Y, Garcia-Jacas CR, Martinez-Mayorga K, Barigye SJ, Vaz d'Almeida YS et al (2017) QuBiLS-MAS, open source multiplatform software for atom- and bond-based topological (2D) and chiral (2.5D) algebraic molecular descriptors computations. J Cheminform 9(1):35

58. Barigye SJ, Marrero-Ponce Y, Alfonso-Reguera V, Pérez-Giménez F (2013) Extended GT-STAF information indices based on Markov approximation models. Chem Phys Lett 570(570):147-152

59. Ruiz-Blanco YB, Paz W, Green J, Marrero-Ponce Y (2015) ProtDCal: A program to compute general-purpose-numerical descriptors for sequences and 3D-structures of proteins. BMC Bioinform 16(1):162

\section{Submit your manuscript to a SpringerOpen ${ }^{\circ}$ journal and benefit from:}

- Convenient online submission

- Rigorous peer review

- Open access: articles freely available online

- High visibility within the field

- Retaining the copyright to your article

Submit your next manuscript at $\boldsymbol{\nabla}$ springeropen.com 